\title{
DICKSON-STIRLING NUMBERS
}

\author{
by L. C. HSU, GARY L. MULLEN* and PETER JAU-SHYONG SHIUE
}

(Received 29th March 1995; revised 12th August 1996)

The Dickson polynomial $D_{n}(x, a)$ of degree $n$ is defined by $D_{n}(x, a)=\sum_{i=0}^{\lfloor n / 2\rfloor} \frac{n}{n-i}\left(\begin{array}{c}n-i \\ i\end{array}\right)(-a)^{i} x^{n-2\rfloor}$, where \lfloor\rfloor denotes the greatest integer function. In particular, we define $D_{0}(x, a)=2$ for all real $x$ and $a$. By using Dickson polynomials we present new types of generalized Stirling numbers of the first and second kinds. Some basic properties of these numbers and a combinatorial application to the enumeration of functions on finite sets in terms of their range values is also given.

1991 Mathematics subject classification: 05A10, 11B65, 11 T06.

\section{Introduction}

The Stirling numbers $s(n, k)$ and $S(n, k)$ of the first and second kinds are usually defined as follows. For $n=1,2, \ldots$, consider the falling factorial

$$
(x)_{n}=x(x-1)(x-2) \ldots(x-n+1)
$$

with $(x)_{0}=1$. Let

$$
\begin{aligned}
& (x)_{n}=\sum_{k=0}^{n} s(n, k) x^{k}, n=0,1, \ldots \\
& x^{n}=\sum_{k=0}^{n} S(n, k)(x)_{k}, n=0,1, \ldots,
\end{aligned}
$$

The coefficients $s(n, k)$ are Stirling numbers of the first kind and are used to express falling factorials in terms of powers of $x$. Similarly the coefficients $S(n, k)$ are called Stirling numbers of the second kind and are used to express powers of $x$ in terms of falling factorials.

There are many properties of Stirling numbers as well as combinatorial applications, see for example Berge [1, pp. 21-22, 37-42] or Straight [16, Chps. 1-2]. We also refer to Charalambides and Singh [3] for a recent survey of properties of Stirling numbers as

* This author would like to thank the National Security Agency for partial support under grant agreement \#MDA904-92-H-3044. 
well as generalizations. See Broder [2], Comtet [4], Hsu [10], and Loeb [13] for other generalizations of the Stirling numbers.

In this paper we will present two new kinds of generalized Stirling numbers, called Dickson-Stirling numbers of the first and second kinds. Some basic properties of these numbers and a combinatorial application will be investigated.

For any fixed real number $a$ consider the Dickson polynomial $D_{n}(x, a)$ of degree $n \geq 1$ defined by

$$
D_{n}(x, a)=\sum_{i=0}^{|n / 2|} \frac{n}{n-i}\left(\begin{array}{c}
n-i \\
i
\end{array}\right)(-a)^{i} x^{n-2 i}
$$

where \lfloor\rfloor denotes the greatest integer function. In particular, we define $D_{0}(x, a)=2$ for all real $x$ and $a$. As indicated in Lidl, Mullen and Turnwald [12], Dickson polynomials enjoy many algebraic and number-theoretic properties. In addition, they play very important roles in the theory of permutation polynomials over finite fields, as well as having numerous applications in various areas including cryptography, pseudoprimality testing, and the construction of irreducible polynomials and normal bases over finite fields. Dickson polynomials are also related to determinants of circulant matrices, see for example Lidl, Mullen and Turnwald [12, p. 178$]$.

Analogous to (1), for a real number $a$ let

$$
(x \mid a)_{n}=(x-a)(x-a-1) \ldots(x-a-n+1),
$$

with $(x \mid a)_{0}=1$. Define $s(n, k ; a)$ and $S(n, k ; a)$, the Dickson-Stirling numbers of the first and second kinds, by

$$
\begin{array}{ll}
(x \mid a)_{n}=\sum_{k=0}^{n} s(n, k ; a)\left(D_{k}(x, a)-c_{k}\right), & n=0,1, \ldots, \\
D_{n}(x, a)-c_{n}=\sum_{k=0}^{n} S(n, k ; a)(x \mid a)_{k}, & n=0,1, \ldots,
\end{array}
$$

where $c_{0}=1$ and $c_{k}=0$ for $k \geq 1$. Obviously $s(0,0 ; a)=S(0,0 ; a)=1$ and one may also define $s(n, k ; a)=S(n, k ; a)=0$ for $n<k$.

Equations (6) and (7) indicate that the Dickson-Stirling numbers can be used to convert between the generalized falling factorial with parameter $a$ defined in (5) and Dickson polynomials with parameter $a$ defined in (4). In particular, when $a=0$, we see that $D_{n}(x, 0)=x^{n}$ for $n \geq 1, D_{0}(x, 0)-c_{0}=1$, and that (6) and (7) reduce to (2) and (3), and so we obtain the usual Stirling numbers of the first and second kinds. When $a=-1,(6)$ and (7) are related to the Lucas polynomials $V_{n}(x)$ (see Filipponi and Horadam [7]) since in this case we have $(x \mid-1)_{n}=(x+1)_{n}$ and thus

$$
D_{n}(x,-1)=\sum_{i=0}^{\lfloor n / 2\rfloor} \frac{n}{n-i}\left(\begin{array}{c}
n-i \\
i
\end{array}\right) x^{n-2 j}=V_{n}(x),(x+1)_{n}=\sum_{k=0}^{n} s(n, k ;-1)\left(V_{k}(x)-c_{k}\right),
$$


and $V_{n}(x)=\sum_{k=0}^{n} S(n, k ;-1)(x+1)_{k}$ for $n \geq 1$.

In Section 2 we develop a number of properties of Dickson-Stirling numbers and in Section 3 we consider various combinatorial sums involving $D_{n}(x, a)$ for $x$ ranging over an interval $\left[m_{1}, m_{2}\right]$, with $0 \leq m_{1}<m_{2}$. Finally in Section 4 we present an application of Dickson-Stirling numbers to the problem of enumerating functions from one finite set to another with various restrictions on the range of the function. This application then reduces for $a=0$ to the usual enumeration via Stirling numbers of functions from one finite set to another.

\section{Dickson-Stirling numbers}

For a polynomial $f(x)$, let $\Delta$ denote the difference operator defined by $\Delta f(x)=$ $f(x+1)-f(x)$. By induction on $k$ we have that $\Delta^{k}(x f(x))=x \Delta^{k} f(x)+k \Delta^{k-1} f(x+1)$. By using Newton interpolation, see Milne-Thomson [14, p. 57], we may write for $n \geq 1$

$$
S(n, k ; a)=\left.\frac{1}{k !} \Delta^{k} D_{n}(x, a)\right|_{x=a} .
$$

From Lidl, Mullen and Turnwald [12, Lemma 2.3] the Dickson polynomials are known to satisfy the recurrence

$$
D_{n+2}(x, a)=x D_{n+1}(x, a)-a D_{n}(x, a), \quad n=0,1, \ldots,
$$

with $D_{0}(x, a)=2$ and $D_{1}(x, a)=x$. Combining these results we have for $n \geq 1$

$$
S(n+2, k ; a)=\left.\frac{1}{k !} \Delta^{k}\left(x D_{n+1}(x, a)\right)\right|_{x=a}-a S(n, k ; a) .
$$

Also

$$
\begin{gathered}
\left.\frac{1}{k !} \Delta^{k}\left(x D_{n+1}(x, a)\right)\right|_{x=a}=\left.\frac{1}{k !} x \Delta^{k} D_{n+1}(x, a)\right|_{x=a}+\left.\frac{1}{(k-1) !} \Delta^{k-1} D_{n+1}(x, a)\right|_{x=a+1} \\
=a S(n+1, k ; a)+\frac{1}{(k-1) !} \Delta^{k} D_{n+1}(a, a)+S(n+1, k-1 ; a) \\
=(a+k) S(n+1, k ; a)+S(n+1, k-1, a) .
\end{gathered}
$$

Hence we have the following recurrence for the Dickson-Stirling numbers of the second kind.

Proposition 1. For $n=0,1, \ldots$ and $k=1,2, \ldots$

$$
S(n+2, k ; a)=(a+k) S(n+1, k ; a)+S(n+1, k-1 ; a)-a S(n, k ; a),
$$


where the initial conditions are

$$
\begin{aligned}
& S(0,0 ; a)=1 \text { and } S(n, 0 ; a)=D_{n}(a, a) \text { for } n>0 \\
& S(n, n ; a)=1 \text { for } n>0 \text { and } S(n, k ; a)=0 \text { for } n<k .
\end{aligned}
$$

Since $S(n, k ; 0)=S(n, k)$, we have the well-known result, see Straight [16, Thm. 2.4.1]:

Corollary 2. For $n=1,2, \ldots$

$$
S(n+2, k)=k S(n+1, k)+S(n+1, k-1) .
$$

We next obtain a generating function for the Dickson-Stirling numbers $S(n, k ; a)$. First, we recall that

$$
\sum_{n=0}^{\infty} D_{n}(x, a) t^{n}=\frac{2-x t}{1-x t+a t^{2}}
$$

see Lidl, Mullen and Turnwald [12, Lemma 2.4]

Proposition 3. For $k=1,2, \ldots$

$$
\sum_{n=k}^{\infty} S(n, k ; a) t^{n}=\frac{1-a t^{2}}{1-a t+a t^{2}} \prod_{j=1}^{k} \frac{t}{1-(a+j) t+a t^{2}}
$$

Also for $k=0$

$$
\sum_{n=0}^{\infty} S(n, 0 ; a) t^{n}=\frac{1-a t^{2}}{1-a t+a t^{2}}
$$

Proof. Denote $F_{k}(t)=\sum_{n=k}^{\infty} S(n, k ; a) t^{n}$. We have

$$
\begin{aligned}
F_{k}(t) & =\sum_{n=k}^{\infty}[S(n-1, k-1 ; a)+(a+k) S(n-1, k ; a)-a S(n-2, k ; a)] t^{n} \\
& =t F_{k-1}(t)+(a+k) t F_{k}(t)-a t^{2} F_{k}(t) .
\end{aligned}
$$

It follows that

$$
F_{k}(t)=\frac{t}{1-(a+k) t+a t^{2}} F_{k-1}(t), \quad k=1,2, \ldots
$$


By successive applications of the above relations we get

$$
F_{k}(t)=F_{0}(t) \prod_{j=1}^{k} \frac{t}{1-(a+j) t+a t^{2}} .
$$

Here $F_{0}(t)$ may be determined as follows via (7) and (9),

$$
\begin{aligned}
F_{0}(t) & =1+\sum_{n=1}^{\infty} S(n, 0 ; a) t^{n} \\
& =1+\sum_{n=1}^{\infty} D_{n}(a, a) t^{n}=-1+\sum_{n=0}^{\infty} D_{n}(a, a) t^{n} \\
& =\frac{2-a t}{1-a t+a t^{2}}-1=\frac{1-a t^{2}}{1-a t+a t^{2}} .
\end{aligned}
$$

This proves both (10) and (11).

Clearly for $a=0$ the proposition gives the generating function for the Stirling numbers of the second kind

$$
\sum_{n=k}^{\infty} S(n, k) t^{n}=\frac{t^{k}}{(1-t)(1-2 t) \ldots(1-k t)}
$$

see Comtet [5, Ch. 5, Thm. C].

As above let $F_{k}(t)$ denote the left hand side of (10) so that

$$
F_{k}(t)=\frac{t}{1-(a+k) t+a t^{2}} F_{k-1}(t)=\frac{t}{1-(a+k) t+a t^{2}} \sum_{m=k-1}^{\infty} S(m, k-1 ; a) t^{m} .
$$

Upon solving the system $\alpha+\beta=a+k$ and $\alpha \beta=a$ for $\alpha$ and $\beta$, we find

$$
\alpha=\frac{1}{2}\left(a+k+\sqrt{(a+k)^{2}-4 a}\right), \quad \beta=\frac{1}{2}\left(a+k-\sqrt{(a+k)^{2}-4 a}\right),
$$

and hence $1-(a+k) t+a t^{2}=(1-\alpha t)(1-\beta t)$ and

$$
\begin{aligned}
\frac{t}{1-(a+k) t+a t^{2}} & =\frac{t}{(1-\alpha t)(1-\beta t)} \\
& =\frac{1}{\alpha-\beta}\left(\frac{1}{1-\alpha t}-\frac{1}{1-\beta t}\right) \\
& =\frac{1}{\alpha-\beta} \sum_{j=1}^{\infty}\left(\alpha^{j}-\beta^{j}\right) t^{j} .
\end{aligned}
$$


Thus

$$
\begin{aligned}
\sum_{n=k}^{\infty} S(n, k ; a) t^{n} & =\frac{1}{\alpha-\beta}\left\{\sum_{j=1}^{\infty}\left(a^{j}-\beta^{j}\right) t^{j}\right\}\left\{\sum_{m=k-1}^{\infty} S(m, k-1 ; a) t^{m}\right\} \\
& =\frac{1}{\alpha-\beta} \sum_{n=k}^{\infty} t^{n} \sum_{j=1}^{n-k+1}\left(\alpha^{j}-\beta^{j}\right) S(n-j, k-1 ; a) .
\end{aligned}
$$

Comparing the coefficient of the term $t^{n}$ we obtain

$$
S(n, k ; a)=\frac{1}{\alpha-\beta} \sum_{j=1}^{n-k+1}\left(\alpha^{j}-\beta^{j}\right) S(n-j, k-1 ; a), \quad(\alpha \neq \beta)
$$

When $a=0$ this reduces to [5, Thm. B, (3d)]:

$$
S(n, k)=\frac{1}{k} \sum_{j=1}^{n-k+1} k^{j} S(n-j, k-1)=\sum_{\lambda=k}^{n} S(\lambda-1, k-1) k^{n-\lambda}
$$

The following simple idea provides a relation between the Dickson-Stirling numbers and the ordinary Stirling numbers of the second kind.

Proposition 4. For given integers $n$ and $k$ with $n \geq k \geq 1$ and a real number $a$

$$
S(n, k ; a)=\sum_{i=0}^{\lfloor(n-k) / 2\rfloor} \sum_{j=0}^{n-2 i-k} \frac{n}{n-i}\left(\begin{array}{c}
n-i \\
i
\end{array}\right)(-1)^{i} a^{i+j}\left(\begin{array}{c}
n-2 i \\
j
\end{array}\right) S(n-2 i-j, k) .
$$

Proof. We have

$$
\begin{aligned}
S(n, k ; a) & =\left.\frac{1}{k !} \Delta^{k} D_{n}(x, a)\right|_{x=a} \\
& =\left.\frac{1}{k !} \sum_{i=0}^{\lfloor n / 2\rfloor} \frac{n}{n-i}\left(\begin{array}{c}
n-i \\
i
\end{array}\right)(-a)^{i} \Delta^{k} x^{n-2 i}\right|_{x=a} \\
& =\sum_{i=0}^{\lfloor n / 2\rfloor} \frac{n}{n-i}\left(\frac{n-i}{i}\right)(-a)^{i}\left\{\sum_{j=0}^{n-2 i}\left(\begin{array}{c}
n-2 i \\
j
\end{array}\right) a^{j} S(n-2 i-j, k)\right\} \\
& =\sum_{i=0}^{\lfloor n / 2\rfloor} \sum_{j=0}^{n-2 i-k} \frac{n}{n-i}\left(\begin{array}{c}
n-i \\
i
\end{array}\right)(-1)^{i} a^{i+j}\left(\begin{array}{c}
n-2 i \\
j
\end{array}\right) S(n-2 i-j, k),
\end{aligned}
$$


from which Proposition 4 follows.

Analogously using the fact that

$$
\left(\begin{array}{c}
n-i \\
i
\end{array}\right)\left(\begin{array}{c}
n-2 i \\
j
\end{array}\right)=\left(\begin{array}{c}
n-i \\
i+j
\end{array}\right)\left(\begin{array}{c}
i+j \\
i
\end{array}\right)
$$

we have with $i+j=r$,

$$
S(n, k ; a)=\sum_{r=0}^{n-k} \sum_{i=0}^{r}(-1)^{i} \frac{n}{n-i}\left(\begin{array}{c}
n-i \\
r
\end{array}\right)\left(\begin{array}{l}
\eta \\
i
\end{array}\right) a^{r} S(n-i-r, k) .
$$

More explicitly

$$
S(n, k ; a)=\sum_{r=0}^{n-k} \sum_{i=0}^{r} \sum_{j=0}^{k} \frac{(-1)^{i+j}}{k !} \frac{n}{n-i}\left(\begin{array}{c}
n-i \\
r
\end{array}\right)\left(\begin{array}{l}
r \\
i
\end{array}\right)\left(\begin{array}{c}
k \\
j
\end{array}\right) a^{r}(k-j)^{n-i-r} .
$$

From (6) and (7) we have

$$
\begin{aligned}
D_{n}(x, a)-c_{n} & =\sum_{k=0}^{n} S(n, k ; a)\left(\sum_{i=0}^{k} s(k, i ; a)\left(D_{i}(x, a)-c_{i}\right)\right) \\
& =\sum_{k=0}^{n} S(n, k ; a)\left(\sum_{i=0}^{n} s(k, i ; a)\left(D_{i}(x, a)-c_{i}\right)\right) \\
& =\sum_{i=0}^{n}\left(\sum_{k=0}^{n} S(n, k ; a) s(k, i ; a)\right)\left(D_{i}(x, a)-c_{i}\right)
\end{aligned}
$$

which yields part (i) of the following result. Part (ii) follows analogously.

Proposition 5. For given non-negative integers $n$ and $i$ and a real number $a$,

(i) $\sum_{k=0}^{n} S(n, k ; a) s(k, i ; a)=\delta_{n i}$,

(ii) $\sum_{k=0}^{n} s(n, k ; a) S(k, i ; a)=\delta_{n i}$.

The $a=0$ case of Proposition 5 for the ordinary Stirling numbers is of course well known. Let $A=(s(n, k ; a))$ and $B=(S(n, k ; a))$ be matrices of size $n \times n$. Then from Proposition 5 we have $A B=I=B A$. If one of the sequences $\left\{f_{k}(x)\right\}_{k \geq 0}$ and $\left\{g_{k}(x)\right\}_{k \geq 0}$ is given, then clearly the reciprocal relation also holds, i.e.,

$$
f_{n}(x)=\sum_{k=0}^{n} s(n, k ; a) g_{k}(x) \Leftrightarrow g_{n}(x)=\sum_{k=0}^{n} s(n, k ; a) f_{k}(x), \quad n=0,1, \ldots
$$


Dickson-Bell numbers may be defined by

$$
B(n, a)=\sum_{k=0}^{n} S(n, k ; a), n=0,1,2, \ldots
$$

The ordinary Bell number corresponds to the case $a=0$. From the definition, we have

$$
\sum_{k=0}^{n} s(n, k ; a) B(k, a)=1
$$

Let $\mathcal{D}_{n}=n !\left(1-\frac{1}{1 !}+\frac{1}{2 !}-\cdots+(-1)^{n} \frac{1}{n !}\right)$ denote the $n$-th derangement number. We now show that the Dickson-Bell number $B(n,-1)$ may be expressed in terms of the derangement numbers $\mathcal{D}_{n}$. To this end we begin by noting that the Lucas number $V_{n}(j)=D_{n}(j,-1)$ for $n \geq 1$. Moreover since $S(n, 0 ;-1)=0$ (resp. 2), for $n \equiv 1$ (resp. 0 ) $(\bmod 2)$, we have $S(n, 0 ;-1)=2 \delta_{n}$, where $\delta_{n}$ is the parity function which is 0 for odd $n$ and 1 for even $n$. Thus we have after some calculations,

$$
\begin{aligned}
B(n,-1)+2 \delta_{n} & =\sum_{k=0}^{n} S(n, k ;-1) \\
& =\sum_{j=0}^{n} \sum_{k=j}^{n} \frac{(-1)^{k-j}}{j !(k-j) !} V_{n}(j) \\
& =\sum_{j=0}^{n} \frac{V_{n}(j) \mathcal{D}_{n-j}}{j !(n-j) !}
\end{aligned}
$$

As a consequence

$$
B(n,-1)=-2 \delta_{n}+\sum_{j=0}^{n} \frac{V_{n}(j) \mathcal{D}_{n-j}}{j !(n-j) !} .
$$

Using Newton's interpolation formula with divided differences, we may obtain a closed formula for the Dickson-Stirling numbers of the second kind. If $f(x)$ is a polynomial of degree $n \geq 1$, then, as indicated for example in Milne-Thomson [14, Sect. 1.1], $f(x)$ can be expressed in the form

$$
f(x)=f\left(\alpha_{0}\right)+\sum_{k=1}^{n}\left[\alpha_{0} \alpha_{1} \ldots \alpha_{k}\right]\{x \mid \alpha\}_{k}
$$

where $\left[\alpha_{0} \alpha_{1} \ldots \alpha_{k}\right]$ are divided differences recursively defined by

$$
\left[\alpha_{0} \alpha_{1} \ldots \alpha_{k}\right]=\frac{\left[\alpha_{0} \alpha_{1} \ldots a_{k-1}\right]-\left[\alpha_{1} \alpha_{2} \ldots \alpha_{k}\right]}{\alpha_{0}-\alpha_{k}}, k \geq 1
$$


with $\left[\alpha_{0} \alpha_{1}\right]=\left(f\left(\alpha_{0}\right)-f\left(\alpha_{1}\right)\right) /\left(\alpha_{0}-\alpha_{1}\right)$ and $\left[\alpha_{0}\right]=f\left(\alpha_{0}\right)$. Also $\{x \mid \alpha\}_{k}=\left(x-\alpha_{0}\right)\left(x-\alpha_{1}\right) \ldots$ $\left(x-\alpha_{k-1}\right)$. Alternatively using determinants we obtain:

$$
\left[\alpha_{0} \alpha_{1} \ldots \alpha_{n}\right]=\left|\begin{array}{ccccc}
1 & \alpha_{0} & \ldots & \alpha_{0}^{n-1} & f\left(\alpha_{0}\right) \\
1 & \alpha_{1} & \ldots & \alpha_{1}^{n-1} & f\left(\alpha_{1}\right) \\
\vdots & \vdots & & \vdots & \vdots \\
1 & \alpha_{n} & \ldots & \alpha_{n}^{n-1} & f\left(\alpha_{n}\right)
\end{array}\right| /\left|\begin{array}{ccccc}
1 & \alpha_{0} & \ldots & \alpha_{0}^{n-1} & \alpha_{0}^{n} \\
1 & \alpha_{1} & \ldots & \alpha_{1}^{n-1} & \alpha_{1}^{n} \\
\vdots & \vdots & & \vdots & \vdots \\
1 & \alpha_{n} & \ldots & \alpha_{n}^{n-1} & \alpha_{n}^{n}
\end{array}\right| .
$$

Applying this with $f(x)=D_{n}(x, a)(n \geq 1)$ and $\alpha_{i}=a+i$ gives the following via algebraic computations

$$
S(n, k ; a)=\sum_{i=0}^{k} D_{n}(a+i, a) \prod_{0 \leq i<j \leq k}^{k}(j-i)^{-1} .
$$

For $a=0$ if we expand by cofactors along the first row we have

$$
\begin{aligned}
S(n, k) & =\left|\begin{array}{cccc}
1 & \ldots & 1^{k-1} & 1^{n} \\
2 & \ldots & 2^{k-1} & 2^{n} \\
\vdots & & \vdots & \vdots \\
k & \ldots & k^{k-1} & k^{n}
\end{array}\right| /\left|\begin{array}{cccc}
1 & 1^{2} & \ldots & 1^{k} \\
2 & 2^{2} & \ldots & 2^{k} \\
\vdots & \vdots & & \vdots \\
k & k^{2} & \ldots & k^{k}
\end{array}\right| \\
& =\frac{1}{1 ! 2 ! \ldots k !}\left|\begin{array}{cccc}
1 & \ldots & 1^{k-1} & 1^{n} \\
2 & \ldots & 2^{k-1} & 2^{n} \\
\vdots & & \vdots & \vdots \\
k & \ldots & k^{k-1} & k^{n}
\end{array}\right|
\end{aligned}
$$

Proposition 6. Let $p$ be an odd prime. Then for any integers $a$ and $k$ with $1<k<p$ we have $S(p, k ; a) \equiv 0(\bmod p), a=0,1,2, \ldots$ In particular for $a=0$ we have $S(p, k) \equiv 0(\bmod p)$.

Proof. Let $\alpha_{i}=a+i, i=0,1,2, \ldots$ Using Newton's interpolation formula [14, p. 57] for $f(x)=D_{p}(x, a)$, we may express $S(p, k ; a)$ in the form

$$
\begin{aligned}
& S(p, k ; a)=\left|\begin{array}{ccccc}
1 & \alpha_{0} & \ldots & \alpha_{0}^{k-1} & D_{p}\left(\alpha_{0}, a\right) \\
1 & \alpha_{1} & \ldots & \alpha_{1}^{k-1} & D_{p}\left(\alpha_{1}, a\right) \\
\vdots & \vdots & & \vdots & \vdots \\
1 & \alpha_{k} & \ldots & \alpha_{k}^{k-1} & D_{p}\left(\alpha_{k}, a\right)
\end{array}\right| / \prod_{0 \leq i<j \leq k}(j-i) \\
& =\left|\begin{array}{ccccc}
1 & \alpha_{0} & \ldots & \alpha_{0}^{k-1} & D_{p}\left(\alpha_{0}, a\right)-\alpha_{0} \\
1 & \alpha_{1} & \ldots & \alpha_{1}^{k-1} & D_{p}\left(\alpha_{1}, a\right)-\alpha_{1} \\
\vdots & \vdots & & \vdots & \vdots \\
1 & \alpha_{k} & \ldots & \alpha_{k}^{k-1} & D_{p}\left(\alpha_{k}, a\right)-\alpha_{k}
\end{array}\right| / \prod_{0 \leq i<j \leq k}(j-i) .
\end{aligned}
$$


From $\left[12\right.$, p. 16], $D_{p}(x, a) \equiv x(\bmod p)$. Hence $D_{p}\left(\alpha_{j}, a\right)-\alpha_{j} \equiv 0(\bmod p)$. Moreover all of the factors $j-i$ in the product are less than $p$ since $k<p$. Since $S(p, k ; a)$ is an integer, as may be seen from Proposition 4 , we can infer that $S(p, k ; a) \equiv 0(\bmod p)$, which completes the proof.

Next we obtain a result concerning the Dickson-Stirling numbers of the first kind.

Proposition 7. For given integers $n, k$ with $n \geq k \geq 1$ and a real number $a$,

$$
s(n+1, k ; a)=s(n, k-1 ; a)-(n+a) s(n, k ; a)+a s(n, k+1 ; a)
$$

with initial conditions $s(n, 0 ; a)=0,(n>0), s(n, n ; a)=1, n \geq 0$, and $s(n, k ; a)=0$, if $n<k$.

Proof. From (6) we have

$$
(x \mid a)_{n+1}=\sum_{k=1}^{n+1} s(n+1, k ; a) D_{k}(x, a)+s(n+1,0 ; a) .
$$

We have $(x \mid a)_{n+1}=(x \mid a)_{n} x-(x \mid a)_{n}(a+n)$ so that the left hand side of the above equation takes the form

$$
\begin{aligned}
(x \mid a)_{n+1}= & \sum_{k=1}^{n} s(n, k ; a) x D_{k}(x, a)-(a+n) \sum_{k=1}^{n} s(n, k ; a) D_{k}(x, a) \\
& +(x-a-n) s(n, 0 ; a) .
\end{aligned}
$$

Using the recurrence (8) for the Dickson polynomials, we have for $k \geq 1$

$$
x D_{k}(x, a)=D_{k+1}(x, a)+a D_{k-1}(x, a) .
$$

Substituting (13) into (12) and by disregarding the term $(x-a-n) s(n, 0 ; a)=0$, we obtain

$$
\begin{aligned}
& \sum_{k=1}^{n} s(n, k ; a) D_{k+1}(x, a)+a \sum_{k=1}^{n} s(n, k ; a) D_{k-1}(x, a)-(a+n) \sum_{k=1}^{n} s(n, k ; a) D_{k}(x, a) \\
& =\sum_{k=2}^{n+1} s(n, k-1 ; a) D_{k}(x, a)+a \sum_{k=0}^{n-1} s(n, k+1 ; a) D_{k}(x, a)-(a+n) \sum_{k=1}^{n} s(n, k ; a) D_{k}(x, a),
\end{aligned}
$$

from which the proposition follows.

We note however that as a recurrence on $n$, Proposition 7 is not useful as a 
computational tool because of the need to have values of $s(n, m ; a)$ for $m=k-1, k$, and $k+1$. Although for $a=0$ we do obtain the well known result $s(n+1, k)=$ $s(n, k-1)-n s(n, k)$, for the ordinary Stirling numbers of the first kind, see Straight $[16$, p. 285].

\section{Summation rules}

Throughout this section we consider $D_{n}(x, a)$ for positive integer $n$. From (7) we can write

$$
D_{n}(x, a)=\sum_{k=0}^{n} k ! S(n, k ; a)\left(\begin{array}{c}
x-a \\
k
\end{array}\right)
$$

If there is a function $F(m, x)$ with a closed formula

$$
\sum_{x=a}^{m} F(m, x)\left(\begin{array}{c}
x-a \\
k
\end{array}\right)=G(m, k),
$$

then by (14) we have

$$
\sum_{x=a}^{m} F(m, x) D_{n}(x, a)=\sum_{k=0}^{n} k ! S(n, k ; a) G(m, k) .
$$

This is an extension of a summation rule for Stirling numbers of the second kind, see Hsu [9]. We now give several examples of this idea in the setting of Dickson-Stirling numbers of the second kind.

Example 1. Let $F(m, x)=1$ and let $G(m, k)=\sum_{x=a}^{m}\left(\begin{array}{c}x-a \\ k\end{array}\right)=\left(\begin{array}{c}m-a+1 \\ k+1\end{array}\right)$. Then by (16) we have

$$
\sum_{x=a}^{m} D_{n}(x, a)=\sum_{k=0}^{n} k ! S(n, k ; a)\left(\begin{array}{c}
m-a+1 \\
k+1
\end{array}\right) .
$$

For $a=0$ we have the well known formula:

$$
\sum_{x=1}^{m} x^{n}=\sum_{k=0}^{n} k ! S(n, k)\left(\begin{array}{c}
m+1 \\
k+1
\end{array}\right) .
$$

Actually there are many known identities of the form (15) in which $F(m, x)$ may consist of a binomial coefficient or a product of binomial coefficients, see for example Egorychev [6], Gould [8], and Riordan [15]. Consequently, we may find various special summation formulas via (16). The following are three more examples. 
Example 2. Let $F(m, x)=\left(\begin{array}{c}m-x \\ r\end{array}\right)$ where $m$ and $r$ are positive integers. Using the fact that $\sum_{x=a}^{m}\left(\begin{array}{c}m-x \\ r\end{array}\right)\left(\begin{array}{c}x-a \\ k\end{array}\right)=\left(\begin{array}{c}m-a+1 \\ r+k+1\end{array}\right)$, we have by (15) and (16)

$$
\sum_{x=a}^{m-r}\left(\begin{array}{c}
m-x \\
r
\end{array}\right) D_{n}(x, a)=\sum_{k=0}^{n} k ! S(n, k ; a)\left(\begin{array}{c}
m-a+1 \\
r+k+1
\end{array}\right) .
$$

Example 3. For real $\alpha$ and $\beta$, the following identity is known, see Gould $[8$, p. 52, Formula (6.14)]:

$$
\sum_{x=0}^{m}\left(\begin{array}{l}
\alpha \\
x
\end{array}\right)\left(\begin{array}{c}
\beta \\
m-x
\end{array}\right)\left(\begin{array}{l}
x \\
k
\end{array}\right)=\left(\begin{array}{l}
\alpha \\
k
\end{array}\right)\left(\begin{array}{c}
\alpha+\beta-k \\
m-k
\end{array}\right)
$$

Thus we obtain

$$
\sum_{x=a}^{m+a}\left(\begin{array}{c}
\alpha \\
x-a
\end{array}\right)\left(\begin{array}{c}
\beta \\
m+a-x
\end{array}\right)\left(\begin{array}{c}
x-a \\
k
\end{array}\right)=\left(\begin{array}{l}
\alpha \\
k
\end{array}\right)\left(\begin{array}{c}
\alpha+\beta-k \\
m-k
\end{array}\right) .
$$

so that by (15) we then have

$$
\sum_{x=a}^{m+a}\left(\begin{array}{c}
\alpha \\
x-a
\end{array}\right)\left(\begin{array}{c}
\beta \\
m+a-x
\end{array}\right) D_{n}(x, a)=\sum_{k=0}^{n} k ! S(n, k ; a)\left(\begin{array}{l}
\alpha \\
k
\end{array}\right)\left(\begin{array}{c}
\alpha+\beta-k \\
m-k
\end{array}\right) .
$$

For $a=0$ we have

$$
\sum_{x=0}^{m}\left(\begin{array}{l}
\alpha \\
x
\end{array}\right)\left(\begin{array}{c}
\beta \\
m-x
\end{array}\right) x^{n}=\sum_{k=0}^{n} k ! S(n, k)\left(\begin{array}{l}
\alpha \\
k
\end{array}\right)\left(\begin{array}{c}
\alpha+\beta-k \\
m-k
\end{array}\right) .
$$

Recall that $D_{0}(x, 0)-c_{0}=1=x^{0}$ so that the above expression is also true for $n=0$, and we have

$$
\sum_{k=0}^{m}\left(\begin{array}{l}
\alpha \\
x
\end{array}\right)\left(\begin{array}{c}
\beta \\
m-x
\end{array}\right)=\left(\begin{array}{c}
\alpha+\beta \\
m
\end{array}\right)
$$

This is the well known Vandermonde convolution formula, see for example Straight [16, p. 167].

Example 4. The following identity is known from Gould [8, p. 54, Formula (6.28)]:

$$
\sum_{x=0}^{m}\left(\begin{array}{l}
m \\
x
\end{array}\right)^{2}\left(\begin{array}{l}
x \\
k
\end{array}\right)=\left(\begin{array}{c}
m \\
k
\end{array}\right)\left(\begin{array}{c}
2 m-k \\
m-k
\end{array}\right)
$$


This implies that

$$
\sum_{x=a}^{m+a}\left(\begin{array}{c}
m \\
x-a
\end{array}\right)^{2}\left(\begin{array}{c}
x-a \\
k
\end{array}\right)=\left(\begin{array}{c}
m \\
k
\end{array}\right)\left(\begin{array}{c}
2 m-k \\
m
\end{array}\right)
$$

As a consequence we may obtain

$$
\sum_{x=a}^{m+a}\left(\begin{array}{c}
m \\
x-a
\end{array}\right)^{2} D_{n}(x, a)=\sum_{k=0}^{n} k ! S(n, k ; a)\left(\begin{array}{c}
m \\
k
\end{array}\right)\left(\begin{array}{c}
2 m-k \\
m
\end{array}\right) .
$$

In the special case when $a=-1$ we have

$$
\sum_{x=-1}^{m-1}\left(\begin{array}{c}
m \\
x+1
\end{array}\right)^{2} V_{n}(x)=\sum_{k=0}^{n} k ! S(n, k ;-1)\left(\begin{array}{c}
m \\
k
\end{array}\right)\left(\begin{array}{c}
2 m-k \\
m
\end{array}\right),
$$

where $V_{n}(x)$ is the Lucas polynomial of degree $n$ defined at the end of Section 1.

\section{A combinatorial application}

In this section we very briefly present a combinatorial application of equation (7), and also of the Dickson-Stirling numbers $S(n, k ; a)$ of the second kind. For $n \geq 1$ and $m \geq 1$, we begin by letting $Z_{n}=\{1,2, \ldots, n\}$ and similarly $Z_{m}=\{1,2, \ldots, m\}$. Then as discovered by Stirling, both sides of equation (3) with $x=m$ count the number of functions from $Z_{n}$ to $Z_{m}$. We now generalize this result by considering the integer parameter $a$ in (7), again with $x=m$. By range or value set of a function $f$ we mean the set $\left\{f(c) \mid c \in Z_{n}\right\}$.

In what follows, $a$ is always assumed to be a non-negative integer less than $m$. For $n \geq 1$, we have from (7) and (4)

$$
\begin{aligned}
D_{n}(m, a)-S(n, 0 ; a) & =D_{n}(m, a)-D_{n}(a, a) \\
& =\sum_{k=0}^{|n / 2|}(-1)^{k} \frac{n}{n-k}\left(\begin{array}{c}
n-k \\
k
\end{array}\right) a^{k}\left(m^{n-2 k}-a^{n-2 k}\right) .
\end{aligned}
$$

Here the coefficient $\frac{n}{n-k}\left(\begin{array}{c}n-k \\ k\end{array}\right)$, as given by "Kaplansky's Cycle Theorem", just represents the number of ways of selecting $k$ objects, with no two consecutive, from $n$ objects arranged in a cycle, see Kaplansky [11]. We will now sketch a proof of the following result using Dickson-Stirling numbers of the second kind. The idea is very similar to that applied to the equation (3) with $x=m$.

Proposition 8. Let $m \geq 1, n \geq 1$ and $0 \leq a<m$ be integers. Then $D_{n}(m, a)-S(n, 0 ; a)$ counts the number of functions $f: Z_{n} \rightarrow Z_{m}$ that take at least one 
integer of the set $\{a+1, a+2, \ldots, m\}$, and such that no integer of $Z_{a}$ occurs consecutively as an image in the cyclic sequence $f(1), f(2), \ldots, f(n), f(1)$.

Proof. The proof essentially follows from (7) with $x=m$ and the fact that for each $1 \leq k \leq n$, the term $S(n, k ; a)(m \mid a)_{k}$ counts the number of functions $f: Z_{n} \rightarrow Z_{m}$ whose range consists of exactly $k$ distinct integers of the set $\{a+1, a+2, \ldots, m\}$ and has the property that no range value $\leq a$ occurs consecutively within the cyclic sequence $f(1), f(2), \ldots, f(n), f(1)$. Thus upon summing from $k=1$ to $k=n$ in (7), the proposition follows since we are thus counting all functions with this property.

An alternative proof can be obtained by using (18) and the Principle of InclusionExclusion.

Since for $a=0$, there are no elements $\leq a$ in the range of any function $f$, we immediately get the trivial case $D_{n}(m, 0)=m^{n}$ that counts the number of functions $f: Z_{n} \rightarrow Z_{m}$.

In Proposition 8 the most important values of $a$ are of course those where $0 \leq a<m$. It may be worth mentioning several related cases which arise from the above proof.

1. If $a=0$ and $k=n, S(n, n ; 0)(m)_{n}=m(m-1) \ldots(m-n+1)$ counts the number of injective functions from $Z_{n}$ to $Z_{m}$.

2. If $a=0$ and $1 \leq k \leq n, S(n, k ; 0)(m)_{k}$ counts the number of functions from $Z_{n}$ to $Z_{m}$ with range of cardinality exactly $k$.

3. If $0 \leq a<m$ and $1 \leq k \leq n, S(n, k ; a)(m \mid a)_{k}$ counts the number of functions from $Z_{n}$ to $Z_{m}$ that take exactly $k$ distinct values in $Z_{m} \backslash Z_{a}$ and such that no range value $\leq a$ occurs consecutively in the cyclic sequence $f(1), f(2), \ldots, f(n), f(1)$.

4. If $0 \leq a<m$ and $k=n, S(n, n ; a)(m \mid a)_{n}=(m-a)(m-a-1) \ldots(m-a-n+1)$ counts the number of functions from $Z_{n}$ to $Z_{m}$ that take $n$ distinct image values in $Z_{m} \backslash Z_{a}$

Acknowledgement. The authors wish to express their sincere thanks to the referee for very helpful comments and suggestions that led to a considerable improvement of this paper.

\section{REFERENCES}

1. C. Berge, Principles of Combinatorics (Math. in Sci. \& Eng., 72, Academic Press, New York, 1971).

2. A. Z. BRODER, The r-Stirling numbers, Discrete Math. 49 (1984), 241-259.

3. C. A. Charalambides and J. Singh, A review of the Stirling numbers, their generalizations and statistical applications, Comm. Statist. (Theor. Methods) 17 (1988), 2533-2595.

4. L. Comtet, Nombres de Stirling genéraux et fonctions symétriques, C.R. Acad. Sci. Paris, Sér. A 275 (1972), 747-750. 
5. L. CoMteT, Advanced Combinatorics (D. Reidel Publishing Co., Dordrecht, Holland, 1974).

6. G. P. Egorychev, Integral Representation and the Computation of Combinatorial Sums. (Transl. Math. Monographs AMS 59, 1984).

7. P. Filipponi and A. F. Horadam, Second derivative sequences of Fibonacci and Lucas polynomials, Fibonacci Quart. 31 (1993), 194-204.

8. H. W. Gould, Combinatorial Identities (Printing \& Binding Co., Morgantown, WV, 1972).

9. L. C. Hsu, A summation rule using Stirling numbers of the second kind, Fibonacci Quart. 31 (1993), 256-262.

10. L. C. Hsu, Some theorems on Stirling-type pairs, Proc. Edinburgh Math. Soc. 36 (1993), 525-535.

11. I. Kaplansky, Solution of the Problème de Ménages, Bull. Amer. Math. Soc. 49 (1943), 784-785.

12. R. Lidl, G. L. Mullen and G. Turnwald, Dickson Polynomials (Pitman Mono. \& Surveys in Pure \& Appl. Math., 65, Longman Scientific \& Technical, Essex, England, 1993).

13. D. E. LOEB, A generalization of the Stirling numbers, Discrete Math. 103 (1992), 259-269.

14. L. M. MiLne-Thомson, The Calculus of Finite Differences (Glasgow University Press, 1951).

15. J. Riordan, Combinatorial Identities (Wiley, New York, 1968).

16. H. J. Straight, Combinatorics, An Invitation (Brooks/Cole Publishing Co., Pacific Grove, CA, 1993).

Institute of Mathematical Sciences

Dalian University OF TECHNOLOGY

DALIAN 116024

P.R. OF CHINA

Department of Mathematical Sciences

UNIVERSITY OF NEVADA, LAS VEGaS

LAS Vegas, NV 89154-4020 U.S.A.

E-mail address: shiue@nevada.edu
Department OF Mathematics

The Pennsylvania State University

UNIVERSITY PARK, PA 16802 U.S.A.

E-mail address: mullen@math.psu.edu 